\title{
Factors Affecting Dimensional Instability of Alginate Impressions during Immersion in the Fixing and Disinfectant Solutions
}

\author{
Setsuo SAITO, Toshio ICHIMARU and Yoshima ARAKI \\ Department of Dental Materials Science and Technology, \\ Iwate Medical University School of Dentistry \\ 1-3-27, Chuo-dori, Morioka, Iwate 020-8505, Japan
}

Received July 15, 1998/Accepted September 14, 1998

To clarify the factors determining the dimensional stability of alginate impressions during immersion in disinfectant and fixing solution, the weight change of impressions in solutions of glutaraldehyde (GA), $\mathrm{NaClO}, \mathrm{Na}_{2} \mathrm{SO}_{4}, \mathrm{~K}_{2} \mathrm{SO}_{4}, \mathrm{CaCl}_{2}$ and $\mathrm{ZnSO}_{4}$ was measured. In the nonelectrolytic solution, GA, the weight decreased in proportion to concentration, possibly due to the gradient of osmotic pressure between the impression and solution. In monovalent metallic salt solutions the weight change decreased with increased concentration. Especially at lower concentrations the rate of weight loss was high. A chemical action of the solution might also be involved, in addition to the osmotic pressure difference. The weight loss in divalent metallic salt solutions was greater than in monovalent solutions, implicating crosslinking reactions between the impression and solution.

Key words : Alginate impression material, Immersion solution, Weight change

\section{INTRODUCTION}

Alginate hydrocolloid impression material poses problems such as contraction or expansion when immersed in disinfectant or fixing solution. The osmotic pressure ${ }^{1,2)}$ gradient between alginate gel and immersion solution and chemical and electrostatic interactions between alginates and other components have been suggested to be the reasons for this. However, these factors have not yet been quantitatively evaluated.

This study was conducted to clarify these effects based on weight change of impressions for clinical use and volume change of desalted calcium alginate gels in nonelectrolyte, monovalent metallic salt and divalent metallic salt solutions. The osmotic pressure of the solutions and the viscosity of sodium alginate sol in the presence or absence of the other ionic species were measured.

\section{MATERIALS AND METHODS}

\section{Materials}

The materials used in this study are shown in Table 1. Powdered alginate impression material, Hi-technicol ${ }^{\circledR}$ (HTC) was used for the study. Two disinfectant solutions of glutaraldehyde (GA) and sodium hypochlorite and four fixing solutions of sodium sulfate, potassium sulfate, zinc sulfate and calcium chloride were used to prepare the 
Table 1 Materials and reagents used for the study

\begin{tabular}{lccc}
\hline \multicolumn{1}{c}{ Material } & Code & W/P & Batch number \\
\hline $\begin{array}{l}\text { Impression } \\
\text { Hi-technicol }\end{array}$ Reagents & HTC & 2.41 & 180625 \\
$\begin{array}{l}\text { Rlutaraldehyde }(25 \%)^{2} \\
\text { Sodium hypochlorite }{ }^{3}\end{array}$ & GA & ESM3059 \\
Sodium sulfate $^{3}$ & - & $706 \mathrm{~S} 1420$ \\
Potassium sulfate $^{3}$ & - & $301 \mathrm{~A} 3085$ \\
Zinc sulfate $^{3}$ & - & $709 \mathrm{~S} 1769$ \\
Calcium chloride $^{2}$ & - & $206 \mathrm{~N} 1825$ \\
Sodium alginate $^{3}$ & - & LKM2553 \\
\hline${ }^{1}$ GC Corporation, Tokyo, Japan & $407 \mathrm{E} 1714$ \\
${ }^{2}$ Wako Pure Chemical Industries, Ltd., Osaka, Japan \\
${ }^{3}$ Kanto Chemical Co., Inc., Tokyo, Japan
\end{tabular}

immersion solutions for alginate impression. Sodium alginate was used to prepare the calcium alginate gel and to evaluate the effect of coexisting ingredients on the morphology of alginate segments in the solution by measurement of viscosity.

\section{Change in impression weight in solution}

The impression material was mixed at the recommended water-to-powder ratio for 45 seconds and introduced into an acrylic cylinder $20 \mathrm{~mm}$ in diameter and $15 \mathrm{~mm}$ in depth. The cylinder was covered with an acrylic plate under loading and left for 8 minutes. The material was then taken out, weighed on a direct-reading balance to a measuring accuracy of $0.1 \mathrm{mg}$, and then immersed in $100 \mathrm{ml}$ of the test solution for 60 minutes. The solution concentration was adjusted to $1,2,3,4,5$, and $6 \mathrm{wt} \%$ for GA, $\mathrm{Na}_{2} \mathrm{SO}_{4}, \mathrm{~K}_{2} \mathrm{SO}_{4}, \mathrm{ZnSO}_{4}$, and $\mathrm{CaCl}_{2}$, and to $0.1,0.2,0.3,0.4,0.5$, and 0.6 wt\% for $\mathrm{NaClO}$. In addition, $0.5 \mathrm{wt} \% \mathrm{Na}_{2} \mathrm{SO}_{4}, 0.5 \mathrm{wt} \% \mathrm{~K}_{2} \mathrm{SO}_{4}$, and 0.3 and $0.6 \mathrm{wt} \% \mathrm{CaCl}_{2}$ solutions were also prepared. Generally, buffer is added to GA solution for disinfection, but in this study it was not added in order to examine the effect of GA alone on the weight change of alginate. After removal the specimen was wiped with filter paper to remove adsorbed water on the surface and weighed, and the percentage change in weight was subsequently determined $(n=3)$.

\section{Change in calcium alginate gel volume in solutions}

A thin film of calcium alginate gel was produced by reacting sodium alginate sol with $\mathrm{CaCl}_{2}$ solution as follows: $1 \%$ sodium alginate sol on an aluminum plate was soaked in $20 \% \mathrm{CaCl}_{2}$ solution for 2 minutes. The resulting gel was left in this solution for 6 hours so that gelation would proceed sufficiently and then cut into pieces of size 43 $\times 30 \times 1 \mathrm{~mm}$. Prior to the immersion test, the gel was desalted by storage excess distilled water changed several times during the course of more than one day.

The change in gel volume following 20 minutes' immersion, $\Delta \mathrm{V}$, was determined from the buoyancy change, as, 
$\Delta=\left(\mathrm{W}-\mathrm{W}^{\prime}\right) / \rho-\mathrm{V}_{0}$

where $\mathrm{W}$ is weight of the specimen in air, $\mathrm{W}^{\prime}$ weight in solution, $\rho$ specific gravity of solution, and $\mathrm{V}_{0}$ volume of specimen before immersion.

\section{Measurement of osmotic concentration of solutions}

$0.15 \mathrm{ml}$ of each solution was examined by a semi-micro osmometer (Yanaco LID Co., Ltd., Kyoto, Japan) for determination of the freezing point. The osmotic concentration $\mathrm{c}(\mathrm{osmol} / \mathrm{kg})$ of the solution was found from the depression of the freezing point, $\Delta \mathrm{T}_{\mathrm{f}}$, using the expression ${ }^{3,4)}$.

$$
c=\Delta \mathrm{T}_{\mathrm{f}} / \mathrm{K}_{\mathrm{f}}
$$

where $K_{f}$ is the constant of molar depression of freezing point.

\section{Measurements of viscosity of sodium alginate sol}

$0.02 \%$ sodium alginate solutions containing $1 \% \mathrm{GA}, 1$ and $5 \% \mathrm{Na}_{2} \mathrm{SO}_{4}, 1$ and $6 \%$ $\mathrm{K}_{2} \mathrm{SO}_{4}$, and $0.1 \% \mathrm{NaClO}$ were prepared. Eight milliliter of each solution were used in Ostwald viscometry at $25.0^{\circ} \mathrm{C}$. From viscometry, the relative viscosity of sodium alginate solution to ingredient solution was calculated.

\section{RESULTS}

Change in weight of the impression in solutions

Fig. 1 shows the weight change of HTC impressions following immersion in solution for 60 minutes. In deionized water ( $0 \%$ concentration), a weight increase of $4.0 \%$ was observed. Weight change in any one solution was less than in water and was noted to decrease with the solution concentration. In GA solution, the linear decrease was remarkable, but a ca. $2 \%$ weight increase was observed even in the solution contain-

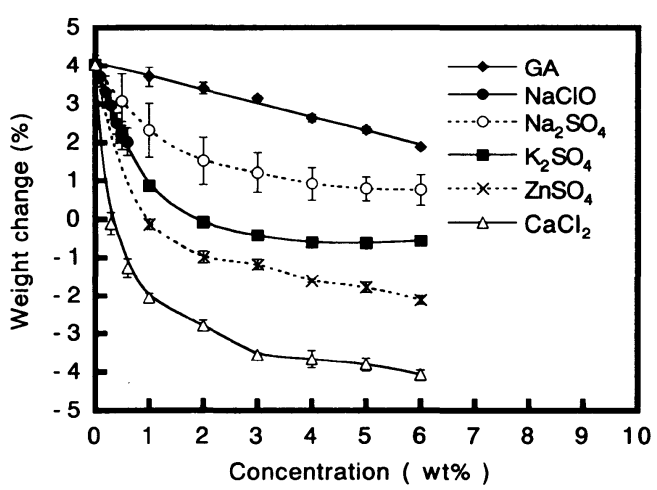

Fig. 1 Weight change of HTC impression after 60 minutes' immersion in solutions.

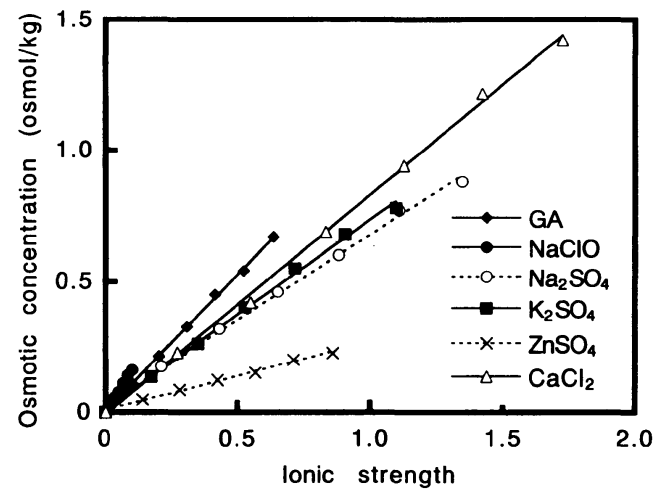

Fig. 2 Relationships between osmotic concentration and ionic strength of solutions. 
ing $6 \% \mathrm{GA}$. In the other solutions, the weight change steeply decreased at concentrations less than $2 \%$, while the decrease was gradual at higher concentrations.

A negative weight change was observed in the solutions of $\mathrm{ZnSO}_{4}, \mathrm{CaCl}_{2}$ and in the above $2 \% \mathrm{~K}_{2} \mathrm{SO}_{4}$ solution. The change was less than $0.1 \%$ in the three solutions $1 \% \mathrm{ZnSO}_{4}, 0.3 \% \mathrm{CaCl}_{2}$, and $2 \% \mathrm{~K}_{2} \mathrm{SO}_{4}$. Weight loss in divalent metallic, $\mathrm{ZnSO}_{4}$ and $\mathrm{CaCl}_{2}$ solutions exceeded that of monovalent metallic salt and nonelectrolyte solutions.

\section{Osmotic concentration of solutions and impression weight change}

Fig. 2 shows the osmotic concentrations of solutions versus ionic strength as determined by the Debye-Hückel second equation, using molar concentration instead of ionic strength only for GA solution. A linear relationship between the osmotic concentration and ionic strength or molar concentration was obtained, as predicted by van't Hoff's law. However, the gradients were lower than 1 . In $\mathrm{ZnSO}_{4}$ solution, the gradient was notably divergent from the theory.

The relationships between weight change of impression and osmotic concentration for each immersion solution are shown in Fig. 3. Similar relationships to those in Fig. 1 were obtained, except that the curve for $\mathrm{ZnSO}_{4}$ was consistent with that for $\mathrm{CaCl}_{2}$, and $\mathrm{NaClO}$ with $\mathrm{Na}_{2} \mathrm{SO}_{4}$.

\section{Change in volume of calcium alginate gel in solution}

Fig. 4 shows the volume change of calcium alginate gel $\left(\Delta V / V_{0}\right)$ when immersed in solution. In GA solution, the gel contracted in proportion to the concentration of the solution. The gel also contracted in $\mathrm{CaCl}_{2}$ and $\mathrm{ZnSO}_{4}$ solutions, more so in the latter than former. In $\mathrm{NaClO}, \mathrm{Na}_{2} \mathrm{SO}_{4}$ and $\mathrm{K}_{2} \mathrm{SO}_{4}$, the increase was $0.37,1.13$ and 1.01 at concentrations of $2.2,2.1$ and $2.6 \mathrm{wt} \%$, respectively. That is, the volume at 20 minutes

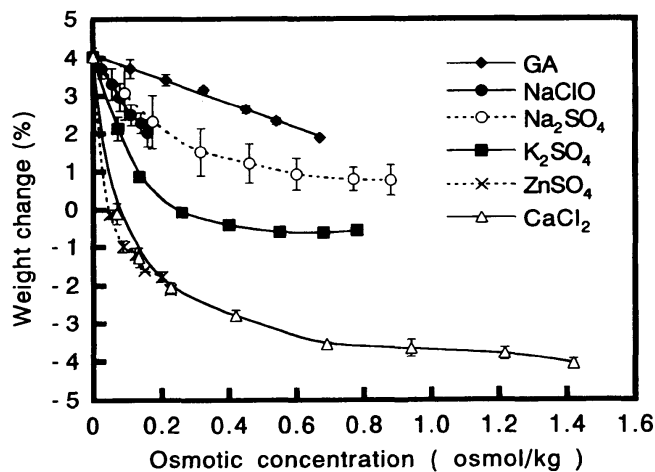

Fig. 3 Relationships between the weight change of HTC impression after 60 minutes' immersion in solutions and the osmotic concentration of the solutions.

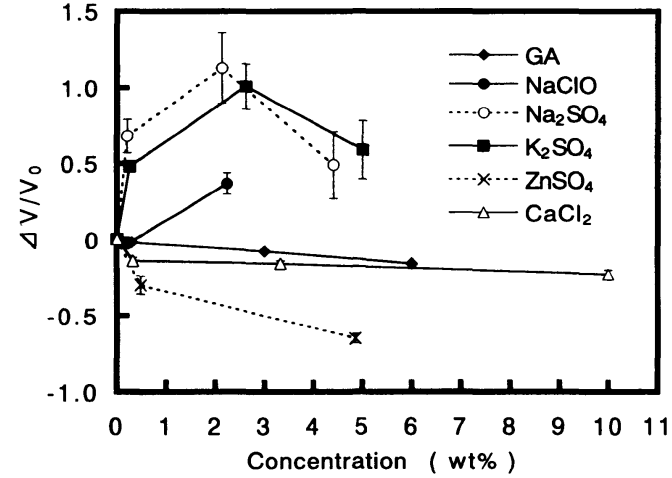

Fig. 4 Relationships between the volume change of calcium alginate gel after 20 minutes' immersion in solutions and the concentration of the solutions. 
Table 2 Relative viscosity of alginate solutions containing various ingredients

\begin{tabular}{lc}
\hline \multicolumn{1}{c}{ Solution } & Relative viscosity $\left(\eta_{\text {rel }}\right)_{25} \mathrm{C}$ \\
\hline $0.02 \%$ Sodium alginate & 2.99 \\
$1 \% \mathrm{GA}-0.02 \%$ Sodium alginate & 1.68 \\
$0.1 \% \mathrm{NaClO}-0.02 \%$ Sodium alginate & 1.30 \\
$1 \% \mathrm{Na}_{2} \mathrm{SO}_{4}-0.02 \%$ Sodium alginate & 1.23 \\
$5 \% \mathrm{Na}_{2} \mathrm{SO}_{4}-0.02 \%$ Sodium alginate & 1.23 \\
$1 \% \mathrm{~K}_{2} \mathrm{SO}_{4}-0.02 \%$ Sodium alginate & 1.22 \\
$6 \% \mathrm{~K}_{2} \mathrm{SO}_{4}-0.02 \%$ Sodium alginate & 1.20 \\
\hline
\end{tabular}

after immersion was 1.37-2.13 times that before immersion. At higher concentrations of $\mathrm{Na}_{2} \mathrm{SO}_{4}$ and $\mathrm{K}_{2} \mathrm{SO}_{4}$ solution, there was a subsequent decrease.

\section{Viscosity of alginate solutions containing the ingredients}

Table 2 shows relative viscosity of alginate solution. This parameter for $0.02 \%$ alginate solution containing no ingredient was 2.99 and less than this value when ingredient was present. Decrease in viscosity with the addition of GA was less than that noted for $\mathrm{NaClO}, \mathrm{Na}_{2} \mathrm{SO}_{4}$ or $\mathrm{K}_{2} \mathrm{SO}_{4}$.

\section{DISCUSSION}

It is well-known that the dimensional change of an alginate impression in disinfectant or fixing solution is due to the movement of the aqueous substance between the impression and solution. The measurement of weight change of an impression was thus conducted in the present study in order to evaluate this parameter and clarify the underlying mechanism. The driving force for the above movement was considered to be the gradient of osmotic pressure between the impression and solution.

The solutions used in this study are classified into three types. The first, GA, is a nonelectrolyte solution. Second, the solutions of $\mathrm{NaClO}, \mathrm{Na}_{2} \mathrm{SO}_{4}$, and $\mathrm{K}_{2} \mathrm{SO}_{4}$ are strong electrolytes having monovalent metallic ions. The third type are solutions of divalent metallic salts, $\mathrm{ZnSO}_{4}$ and $\mathrm{CaCl}_{2}$. Whatever the solution type, the osmotic concentration can be used as a common index for estimating osmotic pressure. However, the weight change of alginate impressions against the osmotic concentration of the immersion solution varied with the solution type, as shown in Fig. 3. This suggested that the osmotic pressure in the impression would vary with the solution type.

In GA solution the degree of swelling was reduced linearly with the osmotic concentration obtained by the freezing point measurement (Fig. 3), and the volume of calcium alginate gel also was decreased with GA concentration (Fig. 4). The linear relationship suggested that the swelling in GA solution is due to the osmotic pressure in GA solution itself, which was expressed as a function of molar concentration of GA as shown in Fig. 2. In turn, the GA solution would have little effect on the osmotic pressure in the impression. 
Weight changes in response to osmotic concentrations of $\mathrm{ZnSO}_{4}$ and $\mathrm{CaCl}_{2}$ solutions were basically the same, as is apparent from Fig. 3, while those against the ionic strength were different as predicted from the relationship between the osmotic concentration and ionic strength shown in Fig. 2. These results suggested that molar concentration, the real solute concentration, was a predominant parameter governing the weight change in these solutions. However, the weight changes in these divalent metallic salt solutions against the osmotic concentration were remarkably lower than in the other two types of solution. Also, the weights decreased with osmotic concentration, resulting in concave curves (Fig. 3). This remarkable weight reduction of impressions in divalent metallic salt solutions may have been due to effects other than those of osmotic pressure. The structure of the alginate gel is composed of a network chain possibly containing unreacted alginate residues. When the impression was immersed in these solutions, the structure should be made stronger through crosslinking reactions between divalent ions and carboxylate ions of alginate. The volume contraction of gel in $\mathrm{ZnSO}_{4}$ and $\mathrm{CaCl}_{2}$ solutions shown in Fig. 4 might be due to the same mechanism. The crosslinking reaction may cause an increase of osmotic pressure in the impression. As stated above, the aqueous substance moves according to the osmotic pressure difference between the impression and the immersion solution. Thus an increase of osmotic pressure in the impression may generate movement of aqueous substance from the gel into the solution, giving the weight decrease of the impression seen in Figs. 1 and 3.

As shown in Fig. 1, the weight changes of impressions in the monovalent metallic $\mathrm{NaClO}, \mathrm{Na}_{2} \mathrm{SO}_{4}$, and $\mathrm{K}_{2} \mathrm{SO}_{4}$ solutions were intermediate between nonelectrolyte and divalent metallic salt solutions. These salts should thus also have effect on the osmotic pressure of a gel in addition to those of the nonelectrolytes. Monovalent metallic ions are not capable of forming crosslinkages with polyanions of alginate. However, it is known that the coexistence of electrolytes in polyelectrolytic solution causes morphology changes to the polymer chain by the electrostatic effect ${ }^{5,6)}$. Viscosity measurement of alginate sol with and without sodium and potassium salts was thus made to examine the effects of coexisting ions on morphological changes of the alginate polymer chain. As shown in Table 2 , the relative viscosity $\left(\eta_{\text {rel }}\right)$ of $0.02 \%$ sodium alginate solution was 2.99, and decreased with the concentration of coexisting ions. This would be reflected by contraction of alginate polymer segments in aqueous media. Thus, in monovalent metallic salt solutions, swelling of the impression may have been reduced due to this contraction in addition to osmotic pressure of the solution. The swelling of desalted gel was noted to increase inversely with the solution concentration (Fig. 4). Differences between components of the impression and desalted gel would perhaps account for this. Partial dissociation of the gel may also have occurred. The effects of monovalent metallic salt solutions on dimensional change should be determined in detail.

Thus the effects of each solution could be summarized as follows:

1) Nonelectrolytic solution: the effect of osmotic pressure

2) Monovalent metallic salt solution: the effect of the chemical action of ions on 
the alginate gel in addition to osmotic pressure effect

3) Divalent metallic salt solution: the effect of crosslinking in addition to those of chemical action and osmotic pressure

The present study has clarified factors that determine dimensional instability of alginate impressions during immersion in each type of solution. Dimensional changes of impressions are due not only to the immersion solution but also to the impression components. In future, the effect of impression components on dimensional instability will need to be examined further.

\section{ACKNOWLEDGMENTS}

The authors wish to thank Dr. Kazuhiko Endo, Department of Dental Materials Science, Health Sciences University of Hokkaido, School of Dentistry for the quantitative analysis of the solution.

\section{REFERENCES}

1) Osada, Y. and Kajiwara, K.: Handbook of gel, 1st ed., N. T.S. Inc., Tokyo, 1997, pp.3947. (in Japanese)

2) Yamauchi, A. and Hirokawa, Y.: Functional gel, 1st ed., Kyoritu Publishing Co., Tokyo, 1996, pp.41-74. (in Japanese)

3) Editorial board of Handbook of chemistry: Handbook of chemistry, 1st ed., Ohm Co., Tokyo, 1978, pp.442-451. (in Japanese)

4) Shimabara, K. and Mitubayashi, H.: Interpretation of chemical structures, 1st ed., Sankyo Publishing Co., Tokyo, 1991, pp.40-54. (in Japanese)

5) Nagasawa, M. and Tatsuzawa, A.: Polymeric water treatment agent, 1st ed., Chijin Publishing Co., Tokyo, 1985, pp.14-42. (in Japanese)

6) Osada, Y. and Kajiwara, K.: Handbook of gel, 1st ed., N. T. S. Inc., Tokyo, 1997, pp.685694. (in Japanese) 